\title{
DETERMINATION OF ALL STABILIZING PI CONTROLLERS FOR LINEAR TIME INVARIANT SYSTEMS
}

\author{
Noha Medhat Mohamed Darwish \\ Electrical Dep., Faculty of Engineering, Assiut University, Assiut, Egypt.
}

Received 15 June 2013; accepted 1 August 2013

\begin{abstract}
This paper considers the problem of controlling a given linear time invariant (LTI) system described by a rational transfer function using a PI controller. A method based on graphical and computational tools has been proposed. This method specifies a PI controller $\left(K_{p}\right.$ and $\left.K_{i}\right)$ that satisfies both frequency domain specifications (a specified gain or phase margin) and minimum integral time absolute error (ITAE).
\end{abstract}

Keywords: ITAE, LTI, Optimal gain and phase margins, PI controller, Robustness, Stabilizing region.

\section{Introduction}

There have been many research works on the tuning of PI and PID controllers because these controllers have simple structures and have been used in industries. [1-2].

Consider a real polynomial $\delta(s)$ of degree $n, \delta(s)$ is Hurwitz if all roots of $\delta(s)=0$ lie in the open left half s-plane $(\operatorname{Re}[\mathrm{s}]<0)$. The problem of checking the stability of the polynomial $\delta(s)$ can be solved using classical criteria such as the Nyquist stability criterion and the Routh-Huriwtz criterion, it is not easy to extend these methods to the cases where PI or PID controllers are involved.

In this paper a simple method in the frequency domain is proposed to tune a PI controller for a LTI system. This method gives another way to determine the stability boundary locus which was presented in [3-6].

In [3-4], the authors, equated the real and the imaginary parts of the characteristic equation in the frequency domain to zero, then they solved both equations to find $K_{p}$ and $K_{i}$ in terms of the frequency variable. They plotted the obtained values of $\left(K_{p}, K_{i}\right)$ in a plane. The determination of the stability boundary was done by choosing a test point within each region.

In [7], the authors gave a computational method for the stabilizing range of $K_{p}$ and $K_{i}$. They used a set of strings that are generated to satisfy specified requirements using the generalized Hermite-Biehler theorem.

In [8], the generalized Mikhailov criterion showed that, the number of roots on the imaginary axis of the characteristic polynomial $\delta(s)$ equals the number of the common zeros of the real part and the imaginary part of the characteristic polynomial $\delta(s)$. The

\footnotetext{
* Corresponding author.

E-mail address: nohamedhat@yahoo.com
} 
Noha Medhat Mohamed Darwish, Determination of All Stabilizing PI Controllers for Linear Time Invariant Systems, pp. 1877 - 1889

system can be marginally stable or unstable depending on the multiplicity of these imaginary roots taking into consideration that no roots are in the RHP.

In [9], the generalized Mikhailov criterion is utilized to give a solution to the problem of finding the stabilizing gains of PI controller for a given LTI plant.

In both [7] and [9], the gains $K_{p}$ and $K_{i}$ are separated to find the stabilizing range. The ranges of $K_{p}$ that satisfy the condition for the existence of a stabilizing $K_{i}$ value at each fixed value of $K_{p}$ was determined by applying the root locus ideas.

In this paper, we propose a simple and fast method to find stability boundary of all stabilizing PI controllers and also those that achieve specified gain and phase margins for a given delay-free LTI plant. This method does not need to apply the root locus ideas as in $[7,9]$ or generate a set of strings as in [7]. The method depends on equating the real and the imaginary parts of the modified characteristic equation in the frequency domain to zero and finding two separate equations for $K_{p}$ and $K_{i}$ in terms of the frequency variable directly without solving equations as in [3-6]. We then compute both stabilizing gains by sweeping over the frequency values algebraically without using a test point as in [3-6].

Gain and phase margins have been used as measures of robustness. A classical approach that takes model uncertainties into consideration is to design the closed-loop control system with sufficient gain and phase margins. We use our proposed method to find all stabilizing PI controllers that achieve a specified gain margin or phase margin.

After determining this stability boundary, we can search for the stabilizing PI controller that gives minimum integral time absolute error (ITAE) for a given plant at the specified gain margin or phase margin. The resulting PI controller will satisfy both robustness and performance requirements.

The specified gain and phase margin in this case are considered as optimal gain and phase margins.

\section{Calculation of all stabilizing PI controllers}

Consider the feedback control system shown in Fig. 1, where

$$
G(s)=\frac{N(s)}{D(s)}
$$

with $N(s), D(s)$ being coprime polynomials and degree $[N(s)] \leq$ degree $[D(s)]$.

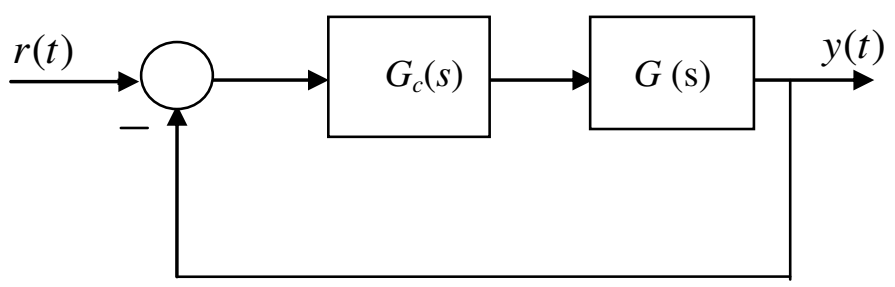

Fig. 1. Feedback control system

Journal of Engineering Sciences, Assiut University, Faculty of Engineering, Vol. 41, No. 5, September, 2013, E-mail address: jes@aun.edu.eg 
Noha Medhat Mohamed Darwish, Determination of All Stabilizing PI Controllers for Linear Time Invariant Systems, pp. 1877 - 1889

The transfer function of PI controller is:

$$
G_{c}(s)=\frac{K_{p} s+K_{i}}{s}
$$

Then the closed-loop characteristic polynomial $\delta(s)$ is:

$$
\delta(s)=s D(s)+\left(K_{i}+K_{p} s\right) N(s)=0
$$

Consider the even-odd decompositions of $N(s)$ and $D(s)$ :

$$
\begin{gathered}
N(s)=N_{e}\left(s^{2}\right)+s N_{o}\left(s^{2}\right), \text { and } \\
D(s)=D_{e}\left(s^{2}\right)+s D_{o}\left(s^{2}\right) .
\end{gathered}
$$

so that

$$
\begin{gathered}
\delta(s)=\left[s^{2} D_{o}\left(s^{2}\right)+s^{2} K_{p} N_{o}\left(s^{2}\right)+K_{i} N_{e}\left(s^{2}\right)\right]+s\left[D_{e}\left(s^{2}\right)+\right. \\
\left.K_{p} N_{e}\left(s^{2}\right)+K_{i} N_{o}\left(s^{2}\right)\right]=0
\end{gathered}
$$

From (5), both the even and the odd parts of $\delta(s)$ depend on $K_{p}$ and $K_{i}$ which causes difficulties when trying to find the ranges of $K_{p}$ and $K_{i}$ that make the system stable. To overcome this problem, we construct a polynomial where the even part of this polynomial depends on $K_{i}$ and the odd part depends on $K_{p}$ [7].

Define:

$$
N^{*}(s)=N(-s)=N_{e}\left(s^{2}\right)-s N_{o}\left(s^{2}\right)
$$

then

$$
\delta(s) N^{*}(s)=\left[s^{2} X_{1}\left(s^{2}\right)+K_{i} X_{2}\left(s^{2}\right)\right]+s\left[X_{3}\left(s^{2}\right)+K_{p} X_{2}\left(s^{2}\right)\right]=0
$$

where

$$
\begin{aligned}
& X_{1}\left(s^{2}\right)=N_{e}\left(s^{2}\right) D_{o}\left(s^{2}\right)-D_{e}\left(s^{2}\right) N_{o}\left(s^{2}\right), \\
& X_{2}\left(s^{2}\right)=N_{e}\left(s^{2}\right) N_{e}\left(s^{2}\right)-s^{2} N_{o}\left(s^{2}\right) N_{o}\left(s^{2}\right), \\
& X_{3}\left(s^{2}\right)=D_{e}\left(s^{2}\right) N_{e}\left(s^{2}\right)-s^{2} D_{o}\left(s^{2}\right) N_{o}\left(s^{2}\right) .
\end{aligned}
$$

Substituting $s=\mathrm{jw}$, we obtain

$$
\delta(j w) N^{*}(j w)=(R(w)+j I(w))\left(N_{R}^{*}(w)+j N_{I}^{*}(w)\right)=U\left(w, K_{i}\right)+j V\left(w, K_{p}\right)=0
$$

where $R(w)$ and $N_{R}^{*}(w)$ are real parts of $\delta(j w)$ and $N^{*}(j w)$ respectively. $I(w)$ and $N_{I}^{*}(w)$ are imaginary parts of $\delta(j w)$ and $N^{*}(j w)$ respectively, and

$$
N_{R}^{*}(w)=N_{e}\left(-w^{2}\right),
$$

Journal of Engineering Sciences, Assiut University, Faculty of Engineering, Vol. 41, No. 5, September, 2013, E-mail address: jes@aun.edu.eg 
Noha Medhat Mohamed Darwish, Determination of All Stabilizing PI Controllers for Linear Time Invariant Systems, pp. 1877 - 1889

$$
\begin{aligned}
N_{I}^{*}(w) & =-w N_{o}\left(-w^{2}\right), \\
U\left(w, K_{i}\right) & =U_{1}(w)+K_{i} U_{2}(w), \\
V\left(w, K_{p}\right) & =V_{1}(w)+K_{p} V_{2}(w), \\
U_{1}(w) & =-w^{2}\left(D_{o}\left(-w^{2}\right) N_{e}\left(-w^{2}\right)-D_{e}\left(-w^{2}\right) N_{o}\left(-w^{2}\right)\right), \\
U_{2}(w) & =N_{e}\left(-w^{2}\right) N_{e}\left(-w^{2}\right)+w^{2} N_{o}\left(-w^{2}\right) N_{o}\left(-w^{2}\right), \\
V_{1}(w) & =w\left(D_{e}\left(-w^{2}\right) N_{e}\left(-w^{2}\right)+w^{2} D_{o}\left(-w^{2}\right) N_{o}\left(-w^{2}\right)\right) .
\end{aligned}
$$

and

$$
V_{2}(w)=w\left(N_{e}\left(-w^{2}\right) N_{e}\left(-w^{2}\right)+w^{2} N_{o}\left(-w^{2}\right) N_{o}\left(-w^{2}\right)\right) .
$$

From these expressions, we note that $K_{i}$ and $K_{p}$ appear in $U\left(w, K_{i}\right)$ and $V\left(w, K_{p}\right)$ respectively.

From (11), we obtain:

$$
U\left(w, K_{i}\right)=0 \text { and } V\left(w, K_{p}\right)=0
$$

So (20) can be rewritten as,

$$
\begin{aligned}
K_{p} & =-\frac{V_{1}(w)}{V_{2}(w)} \\
K_{i} & =-\frac{U_{1}(w)}{U_{2}(w)}
\end{aligned}
$$

Hence, the stabilizing region of $\left(K_{p}, K_{i}\right)$ values can be plotted with w varies from a zero to a finite value $w=w_{0}$. The frequency $w_{0}$ is frequency value of a significant point on the Nyquist plot of the plant where $\angle G\left(j w_{0}\right)= \pm 180^{\circ}$ or $\angle G\left(j w_{0}\right)=0^{\circ}$ (Imaginary part of $G(j w)=0)$.

\subsection{The steps of the proposed method}

1- Initialize $w=0$, step $=0.005$ and $w_{\max }=w_{0}$

2- Compute $K_{p}$ and $K_{i}$ using (21) and (22), then check the stability of the closed-loop system at this point $\left(K_{p}, K_{i}\right)$ by finding the roots of $\delta(s)$.

3 - If the system is stable, find the other positive real value of $w \leq w_{\max }$ at the same $K_{p}$ obtained in step (2) by solving the equation $V\left(w, K_{p}\right)=0$.

4- At this value of $w$ obtained from step (3), compute $K_{i}$ from (22). Check the stability of the closed-loop system. The value of $K_{i}$ where the system is stable will be the second value at the fixed value of $K_{p}$ obtained before in step (2). Since the stabilizing

Journal of Engineering Sciences, Assiut University, Faculty of Engineering, Vol. 41, No. 5, September, 2013, E-mail address: jes@aun.edu.eg 
Noha Medhat Mohamed Darwish, Determination of All Stabilizing PI Controllers for Linear Time Invariant Systems, pp. 1877 - 1889

region is two dimensional space (2D), so for every value of $K_{p}$, there are two values of $K_{i}$, the lower bound $K_{i} \min$ and the upper bound $\boldsymbol{K}_{\boldsymbol{i} \max }$.

5- Increase $w$ as follows: $w=w+$ step

6- If $w \leq w_{\max }$ go to step (2), else stop the method.

We write a MATLAB program to run this algorithm.

\section{All stabilizing PI controllers for specified gain and phase margins}

In this section, we consider the problem of finding all stabilizing PI controllers that achieve specified gain and phase margins for a given LTI plant. Let $2 \leq A_{m} \leq 5$ and $30^{\circ} \leq \theta_{m} \leq 60^{\circ}$ denote the desired gain and phase margin [10].

Consider Fig. 2 with a gain-phase margin tester [11],

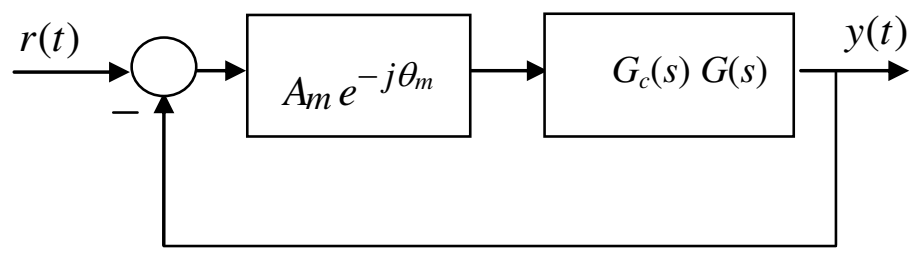

Fig. 2. Feedback control system with gain-phase tester.

Then the characteristic equation can be written as follows:

$$
\delta(s)=s D(s)+A_{m} e^{-j \theta_{m}}\left(K_{i}+K_{p} s\right) N(s)=0
$$

Multiply Eq.(23) by $e^{j \theta_{m}}$, we obtain

$$
\delta(s)=e^{j \theta_{m}} s D(s)+A_{m}\left(K_{i}+K_{p} s\right) N(s)=0
$$

(9) will be modified to the new equation as:

$$
\delta(j w) N^{*}(j w)=U\left(w, K_{i}\right)+j V\left(w, K_{p}\right)=0
$$

where $U\left(w, K_{i}\right)$ and $V\left(w, K_{p}\right)$ are defined in (14) and (15) with new equations of $U_{1}(w), U_{2}(w), V_{1}(w)$, and $V_{2}(w)$ are as follows:

$$
\begin{aligned}
U_{1}(w)= & -w^{2} \cos \left(\theta_{m}\right)\left(D_{o}\left(-w^{2}\right) N_{e}\left(-w^{2}\right)-D_{e}\left(-w^{2}\right) N_{o}\left(-w^{2}\right)\right)-w \sin \left(\theta_{m}\right) \\
& \left.+w^{2} D_{o}\left(-w^{2}\right) N_{o}\left(-w^{2}\right)\right) \\
U_{2}(w)= & A_{m}\left(N_{e}\left(-w^{2}\right) N_{e}\left(-w^{2}\right)+w^{2} N_{o}\left(-w^{2}\right) N_{o}\left(-w^{2}\right)\right),
\end{aligned}
$$


Noha Medhat Mohamed Darwish, Determination of All Stabilizing PI Controllers for Linear Time Invariant Systems, pp. 1877 - 1889

$$
\begin{aligned}
V_{1}(w)= & -w^{2} \sin \left(\theta_{m}\right)\left(D_{o}\left(-w^{2}\right) N_{e}\left(-w^{2}\right)-D_{e}\left(-w^{2}\right) N_{o}\left(-w^{2}\right)\right)+w \cos \left(\theta_{m}\right) \\
& \left(D_{e}\left(-w^{2}\right) N_{e}\left(-w^{2}\right)+w^{2} D_{o}\left(-w^{2}\right) N_{o}\left(-w^{2}\right)\right),
\end{aligned}
$$

and

$$
V_{2}(w)=w A_{m}\left(N_{e}\left(-w^{2}\right) N_{e}\left(-w^{2}\right)+w^{2} N_{o}\left(-w^{2}\right) N_{o}\left(-w^{2}\right)\right) .
$$

The values of $K_{p}$ and $K_{i}$ on the stability boundary can be computed using the (21) and (22).

We have two design specifications to obtain the stability boundary of $\left(K_{p}, K_{i}\right)$ as follows:

For design specification on a specified gain margin $A_{m}$, we set $\theta_{m}=0$ in (26) and (28).

For design specification on a specified phase margin $\theta_{m}$, we set $A_{m}=1$ in (27) and (29).

In both cases, the steps proposed in section 2.1 can now be used to solve this problem. But for the case of the phase margin, the frequency $w_{0}$ satisfies the relation $\angle G\left(j w_{0}\right)=-180^{\circ}+\theta_{m}$ is considered. From this stability boundary, we can search for the stabilizing PI controller that gives minimum ITAE for a given plant, so the optimal gain or phase margin can be achieved.

The ITAE is defined as:

$$
\text { ITAE }=\int_{0}^{\infty} t|e(t)| d t
$$

This optimization is made with the response obtained via simulation using MATLAB program. We compute the unit step response of the closed-loop system for all stabilizing PI controllers that lie on the stability boundary that satisfies the specified $A_{m}$ or $\theta_{m}$, depending on the design specification. Then we compute the error $(\mathrm{e}(\mathrm{t})=\mathrm{y}(\mathrm{t})-\mathrm{r}(\mathrm{t}))$ and applying the trapezoidal rule to approximate the integration in (30). Finally we search for stabilizing point $\left(K_{p}, K_{i}\right)$ that gives minimum value of ITAE.

We choose ITAE since the corresponding response of this criterion tends to have smaller settling time and overshoot than that of the integral squared error (ISE) and the integral absolute error (IAE) because of the time weighting factor in (30).

\section{Numerical Example}

Consider the control system in Fig. 1 with the plant [7],

$$
G(s)=\frac{N(s)}{D(s)}=\frac{s^{4}+6 s^{3}+12 s^{2}+54 s+16}{s^{5}+11 s^{4}+22 s^{3}+60 s^{2}+47 s+25}
$$

The first aim is to find all stabilizing PI controllers that make the characteristic polynomial of (3) Hurwitz.

From (16), (17), (18), and (19) 
Noha Medhat Mohamed Darwish, Determination of All Stabilizing PI Controllers for Linear Time Invariant Systems, pp. 1877 - 1889

$$
\begin{gathered}
U_{1}(w)=-w^{10}-32 w^{8}+627 w^{6}-2474 w^{4}+598 w^{2} \\
U_{2}(w)=w^{8}+12 w^{6}-472 w^{4}+2532 w^{2}+256 \\
V_{1}(w)=5 w^{9}-6 w^{7}-549 w^{5}+1278 w^{3}+400 w \\
V_{2}(w)=w^{9}+12 w^{7}-472 w^{5}+2532 w^{3}+256 w
\end{gathered}
$$

So (21) and (22) will be as follows:

$$
\begin{gathered}
K_{p}=\frac{-5 w^{9}+6 w^{7}+549 w^{5}-1278 w^{3}-400 w}{w^{9}+12 w^{7}-472 w^{5}+2532 w^{3}+256 w}, \\
K_{i}=\frac{w^{10}+32 w^{8}-627 w^{6}+2474 w^{4}-598 w^{2}}{w^{8}+12 w^{6}-472 w^{4}+2532 w^{2}+256},
\end{gathered}
$$

By following the steps presented in section 2.1, where $w \in\left[0, w_{0}\right]$ and $w_{0}=2.4162$ $\mathrm{rad} / \mathrm{sec}$, we can determine the stabilizing region $\left(K_{p}, K_{i}\right)$ as shown in Fig. 3, (The outer curve).

The range of $K_{p}$ for stable system is:

$$
-0.787425<K_{p}<2.484578
$$

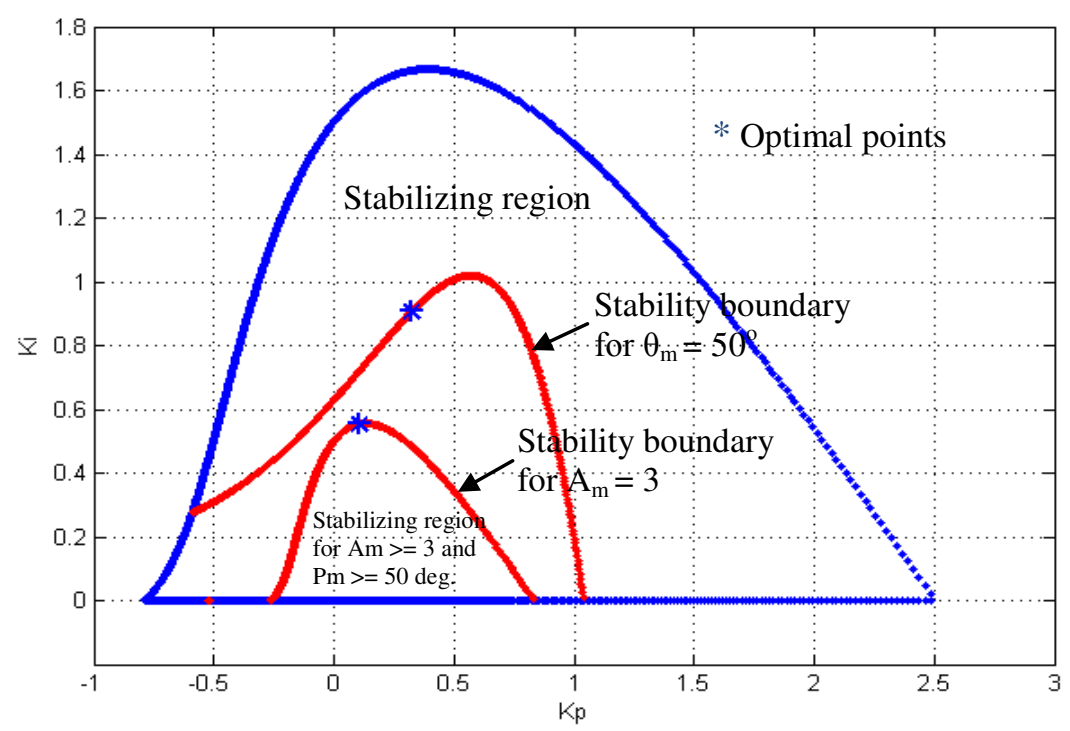

Fig. 3. The stabilizing regions

Journal of Engineering Sciences, Assiut University, Faculty of Engineering, Vol. 41, No. 5, September, 2013, E-mail address: jes@aun.edu.eg 
Noha Medhat Mohamed Darwish, Determination of All Stabilizing PI Controllers for Linear Time Invariant Systems, pp. 1877 - 1889

The second aim is to find all stabilizing PI controllers which satisfy the conditions that the phase margin of the system is greater than $50^{\circ}$ or the gain margin is greater than $3(9.54$ $\mathrm{db}$ ). So we compute the stability boundary using (26), (27), (28), and (29).

1. For $A_{m}=3$ and $\theta_{m}=0$, From (26), (27), (28), and (29)

$$
\begin{gathered}
U_{1}(w)=-w^{10}-32 w^{8}+627 w^{6}-2474 w^{4}+598 w^{2}, \\
U_{2}(w)=3 w^{8}+36 w^{6}-1416 w^{4}+7596 w^{2}+768, \\
V_{1}(w)=5 w^{9}-6 w^{7}-549 w^{5}+1278 w^{3}+400 w \\
V_{2}(w)=3 w^{9}+36 w^{7}-1416 w^{5}+7596 w^{3}+768 w .
\end{gathered}
$$

So (21) and (22) will be as follows:

$$
\begin{gathered}
K_{p}=\frac{-5 w^{9}+6 w^{7}+549 w^{5}-1278 w^{3}-400 w}{3 w^{9}+36 w^{7}-1416 w^{5}+7596 w^{3}+768 w}, \\
K_{i}=\frac{w^{10}+32 w^{8}-627 w^{6}+2474 w^{4}-598 w^{2}}{3 w^{8}+36 w^{6}-1416 w^{4}+7596 w^{2}+768},
\end{gathered}
$$

By following the steps presented in section 2.1, where $w \in[0,2.4162]$ and searching only for $K_{i \max }$, we can draw the stability boundary $\left(K_{p}, K_{i}\right)$ as shown in Fig. 3.

The range of $K_{p}$ for stable system is:

$$
-0.262475<K_{p}<0.828193
$$

After determining this stability boundary, we can search through all the points forming this boundary to find the $\left(K_{p}, K_{i}\right)$ point that gives minimum ITAE. This point is represented by a blue notation * in Fig. 3 .

The resulting PI controller transfer function is:

$$
G_{c}(s)=\frac{K_{p} s+K_{i}}{s}=\frac{0.106633+0.554035}{s}
$$

The bode plot of the open-loop transfer function is shown in Fig. 4. In this case, $A_{m}$ is called optimal gain margin.

The step response of the closed-loop system with minimum ITAE at $A_{m}=3$ is shown in Fig. 5.

Journal of Engineering Sciences, Assiut University, Faculty of Engineering, Vol. 41, No. 5, September, 2013, E-mail address: jes@aun.edu.eg 
Noha Medhat Mohamed Darwish, Determination of All Stabilizing PI Controllers for Linear Time Invariant Systems, pp. 1877 - 1889

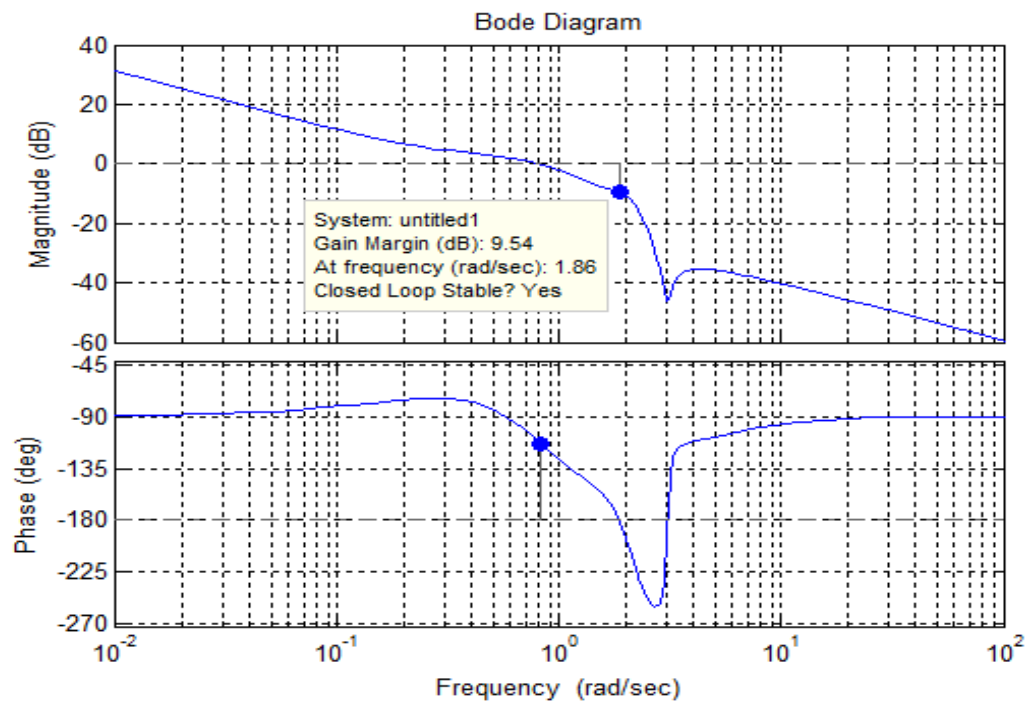

Fig. 4. The bode plot of the open-loop transfer function at optimal $A_{m}=3$.

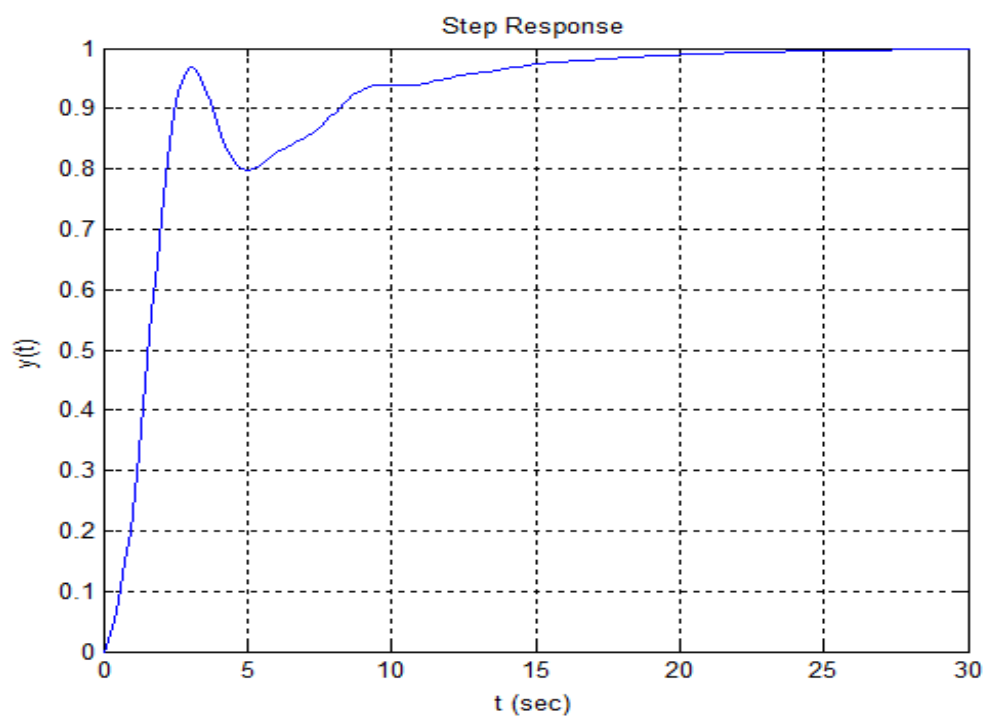

Fig. 5. The step response of the closed-loop system with minimum ITAE at $A_{m}=3$.

Journal of Engineering Sciences, Assiut University, Faculty of Engineering, Vol. 41, No. 5, September, 2013, E-mail address: jes@aun.edu.eg 
Noha Medhat Mohamed Darwish, Determination of All Stabilizing PI Controllers for Linear Time Invariant Systems, pp. 1877 - 1889

2. For $A_{m}=1$ and $\theta_{m}=50^{\circ}$,

From (26), (27), (28), and (29)

$$
\begin{aligned}
& U_{1}(w)=-0.64279 w^{10}-3.8302 w^{9}-20.5692 w^{8}+4.5963 w^{7}+403.0278 w^{6}+420.5584 w^{5} \\
& -1590.2565 w^{4}-979.0048 w^{3}-384.387 w^{2}-306.4178 w, \\
& U_{2}(w)=w^{8}+12 w^{6}-472 w^{4}+2532 w^{2}+256, \\
& V_{1}(w)=-0.76604 w^{10}+3.2139 w^{9}-24.5134 w^{8}-3.8567 w^{7}+480.3099 w^{6}-352.8904 w^{5} \\
& -1895.194 w^{4}+821.4826 w^{3}+458.0946 w^{2}+257.115 w, \\
& V_{2}(w)=w^{9}+12 w^{7}-472 w^{5}+2532 w^{3}+256 w .
\end{aligned}
$$

So (21) and (22) will be as follows:

$$
\begin{gathered}
K_{p}=\frac{\begin{array}{c}
0.76604 w^{10}-3.2139 w^{9}+24.5134 w^{8}+3.8567 w^{7}-480.3099 w^{6}+352.8904 w^{5} \\
+1895.194 w^{4}-821.4826 w^{3}-458.0946 w^{2}-257.115 w
\end{array}}{w^{9}+12 w^{7}-472 w^{5}+2532 w^{3}+256 w} \\
K_{i}=\frac{0.64279 w^{10}+3.8302 w^{9}+20.5692 w^{8}-4.5963 w^{7}-403.0278 w^{6}-420.5584 w^{5}}{+1590.2565 w^{4}+979.0048 w^{3}+384.387 w^{2}+306.4178 w} \\
w^{8}+12 w^{6}-472 w^{4}+2532 w^{2}+256
\end{gathered}
$$

By following the steps presented in section 2.1, and searching only for $K_{i \max }$, we can draw the stability boundary $\left(K_{p}, K_{i}\right)$ as shown in Fig. 3. The range of w needed for stabilization for this case can be found from $\angle G\left(j w_{0}\right)=-130^{\circ}$, so the range will be $w \in[0,2.0201]$.

The range of $K_{p}$ for stable system is:

$$
-0.586993<K_{p}<1.038473
$$

After determining this stability boundary, we can search through all the points forming this boundary to find the $\left(K_{p}, K_{i}\right)$ point that gives minimum ITAE. This point is represented by a blue notation $*$ in Fig. 3 .

The resulting PI controller transfer function is:

$$
G_{c}(s)=\frac{K_{p} s+K_{i}}{s}=\frac{0.324399 s+0.907103}{s}
$$

Journal of Engineering Sciences, Assiut University, Faculty of Engineering, Vol. 41, No. 5, September, 2013,E-mail address: jes@aun.edu.eg 
1887

Noha Medhat Mohamed Darwish, Determination of All Stabilizing PI Controllers for Linear Time Invariant Systems, pp. 1877 - 1889

The bode plot of the open-loop transfer function is shown in Fig. 6. In this case, $\theta_{m}$ is called optimal phase margin. The step response of the closed-loop system with minimum ITAE at $\theta_{m}=50^{\circ}$ is shown in Fig. 7.

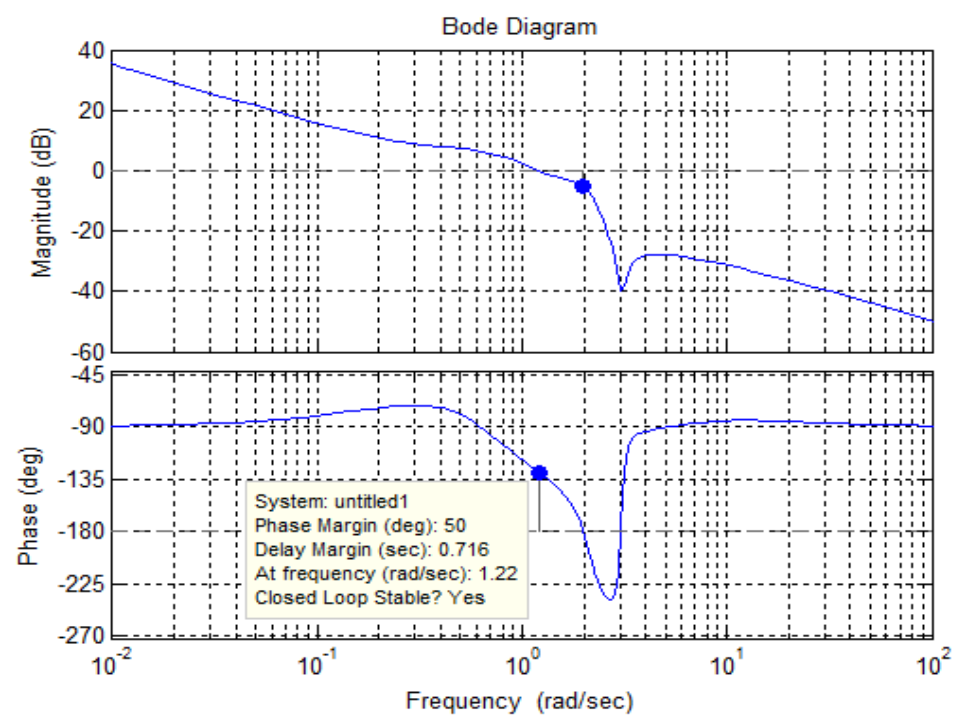

Fig. 6. The bode plot of the open-loop transfer function at optimal $\theta_{m}=50^{\circ}$.

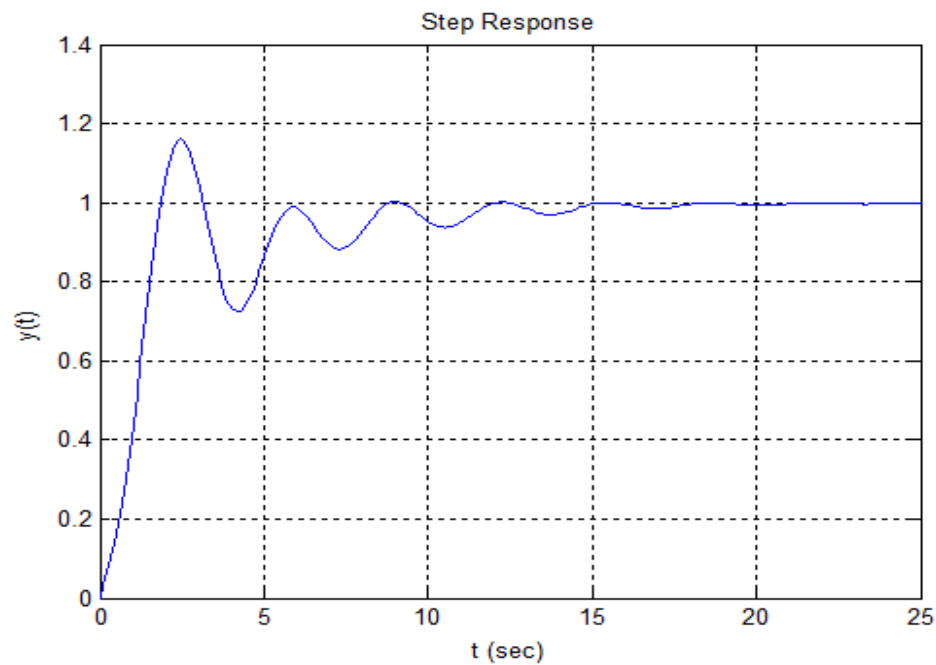

Fig. 7. The step response of the closed-loop system with minimum ITAE at $\theta_{m}=50^{\circ}$.

Journal of Engineering Sciences, Assiut University, Faculty of Engineering, Vol. 41, No. 5, September, 2013, E-mail address: jes@aun.edu.eg 
Noha Medhat Mohamed Darwish, Determination of All Stabilizing PI Controllers for Linear Time Invariant Systems, pp. 1877 - 1889

\section{Conclusions}

In this paper, we proposed a simple method to compute all stabilizing PI gains limits. This method depends on equating the real and the imaginary parts of the modified characteristic equation to zero and finding two separate equations for $K_{p}$ and $K_{i}$. We then compute both gains by sweeping over the frequency values.

By this method, we can also find all stabilizing PI controllers that achieve specified gain and phase margins. From the stability boundary, we find easily the gains of the PI controller that gives optimal gain or phase margin by searching for minimum ITAE. Our future work is to investigate PI/PID controllers with time-delay systems.

\section{References}

[1] K. J. Astrom and T. Hagglund, PID Controllers: Theoy, Design, and Tuning. Instrument Society of America, 1995.

[2] W. K. Ho, C. C. Hang, and L. S. Cao, "Tuning of PID controllers based on gain and phase margins specifications," Automatica, vol. 31, pp. 497-502, 1995.

[3] N. Tan, and I. Kaya, "Computation of stabilizing PI and PID controllers," Proceedings of 2003 IEEE Conference on Control Applications, CCA, vol.2, pp. 876-881, 2003.

[4] N. Tan, I. Kaya, C. Yeroglu and D. P. Atherton "Computation of stabilizing PI and PID controllers using the stability boundary locus," Energy Conversion and Management, vol.47, issues 18-19, pp. 3045-3058, 2006.

[5] N. Tan, "Computation of stabilizing PI-PD controllers," International Journal of Control, Automation, and Systems, vol. 7, no. 2, pp.175-184, 2009.

[6] R. Matusu, R. Prokop, and Z. Prokopova, "Simple Tuning of PI Controllers for Interval Plants," Manuf. and Ind. Eng., (C Faculty of Manuf. Tech. TUKE, issue 11, vol. 2, pp. 44-47, 2012.

[7] G. J. Silva, A. Datta, and S. P. Bhattacharyya, "PID Controllers for Time-Delay Systems," Birkhäuser, Boston, 2005.

[8] A. I. Saleh, M. M. Hasan, and N. M. M. Darwish, "The Mikhailov Stability Criterion Revisited," Journal of Engineering Sciences, Published by Faculty of Engineering-Assiut University-Egypt, vol. 38, no. 1, pp. 195-207, January 2010.

[9] A. I. Saleh, M. M. Hasan, and N. M. M. Darwish, "Determination of Controllers Gains Limit Using The Mikhailov Stability Criterion," Journal of Engineering Sciences, Published by Faculty of Engineering-Assiut University-Egypt, vol. 38, no. 1, pp. 209-219, January 2010.

[10] W. K. HO, K. W. LIM, and W. XU, "Optimal Gain and Phase Margin Tuning for PID Controllers," Automatica, vol. 34, no. 8, pp. 1009-1014, 1998.

[11] C. H. Chang, and K. W. Han, "Gain margins and phase margins for control systems with adjustable parameters," Journal of Guidance, Control, and Dynamics, vol. 13, no. 3, pp. 404408, 1990. 


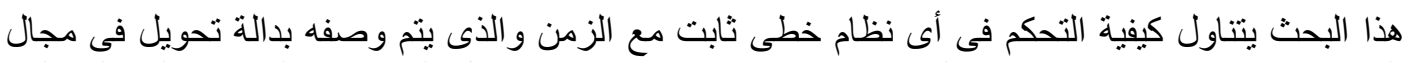

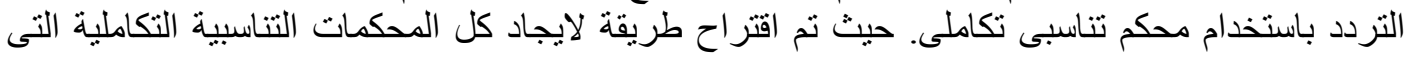

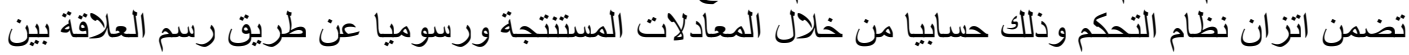

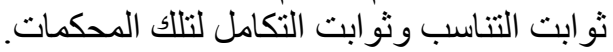

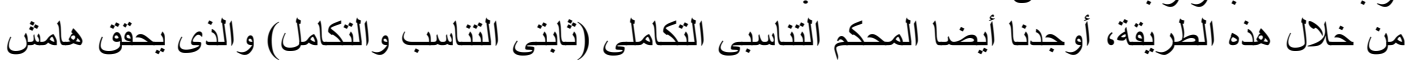

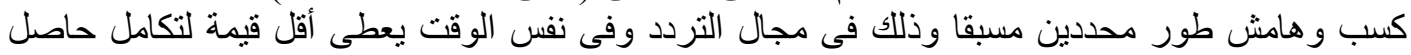

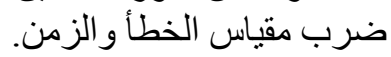
ولقد أعطي مثال توضيحي يبين الطريقة المقترحة لايجاد معاملات المحكمات التناسبية التكاملية والتى التى

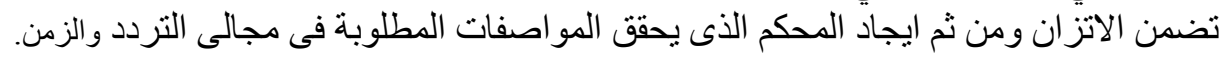

